\title{
NOTES ON \\ TRANSLITERATION \\ AND TRANSLATION
}

I have followed a modified Library of Congress system when transliterating from Russian to English, adapting it when necessary to align with customary English spelling. For example, first and last names ending in -ii have been changed to $-y$, such as Dmitry and Dostoevsky; soft signs have been omitted in names and places. However, all bibliographical references, including endnotes and parenthetical notations, follow the standard Library of Congress system. Unless otherwise noted, all translations from the Russian are my own. 

LOVE FOR SALE 
